\title{
Monitoring SARS-CoV-2 Circulation and Diversity through Community Wastewater Sequencing, the Netherlands and Belgium
}

\author{
Ray Izquierdo-Lara, Goffe Elsinga, Leo Heijnen, Bas B. Oude Munnink, \\ Claudia M.E. Schapendonk, David Nieuwenhuijse, Matthijs Kon, Lu Lu, Frank M. Aarestrup, \\ Samantha Lycett, Gertjan Medema, ${ }^{1}$ Marion P.G. Koopmans, ${ }^{1}$ Miranda de Graaf ${ }^{1}$
}

Severe acute respiratory syndrome coronavirus 2 (SARSCoV-2) has rapidly become a major global health problem, and public health surveillance is crucial to monitor and prevent virus spread. Wastewater-based epidemiology has been proposed as an addition to disease-based surveillance because virus is shed in the feces of $\approx 40 \%$ of infected persons. We used next-generation sequencing of sewage samples to evaluate the diversity of SARS-CoV-2 at the community level in the Netherlands and Belgium. Phylogenetic analysis revealed the presence of the most prevalent clades (19A, 20A, and 20B) and clustering of sewage samples with clinical samples from the same region. We distinguished multiple clades within a single sewage sample by using low-frequency variant analysis. In addition, several novel mutations in the SARS-CoV-2 genome were detected. Our results illustrate how wastewater can be used to investigate the diversity of SARS-CoV-2 viruses circulating in a community and identify new outbreaks.

ince its discovery, severe acute respiratory syn$\checkmark$ drome coronavirus 2 (SARS-CoV-2) has caused $>100$ million confirmed cases of coronavirus disease (COVID-19). The global effects of SARS-CoV-2 and the need to learn more about its origin and epidemiology have resulted in the sequencing of $>416,000$ genomes as of January 2021 (1). This work has enabled the identification of groups of viruses that, on

Author affiliations: Erasmus University Medical Center, Rotterdam, the Netherlands (R. Izquierdo-Lara, B.B. Oude Munnink,

C.M.E. Schapendonk, D. Nieuwenhuijse, M. Kon,

M.P.G. Koopmans, M. de Graaf); KWR Water Research

Institute, Nieuwegein, the Netherlands (G. Elsinga, L. Heijnen,

G. Medema); University of Edinburgh, Edinburgh, Scotland, UK

(L. Lu, S. Lycett); Technical University of Denmark, Kongens

Lyngby, Denmark (F.M. Aarestrup)

DOI: https://doi.org/10.3201/eid2705.204410 the basis of their genetic diversity, can be associated with geographic and temporal patterns of virus spread (2). Nextstrain (https://nextstrain.org) currently divides SARS-CoV-2 diversity into 12 major global clades (19A, 19B, and 20A-20J), on the basis of high prevalence, signature mutations, and geographic spread (3).

Although SARS-CoV-2 primarily affects respiratory tract tissues, it can also replicate in the gastrointestinal tract, as evidenced by in vitro infection of enteroids (4), presence of viral proteins in gastrointestinal epithelium biopsy specimens (5), and detection of infectious virus in stool samples (6). Viral RNA is shed in the feces of $\approx 40 \%$ of infected persons, often for longer periods than the virus can be detected in nasal swab specimens. SARS-CoV-2 RNA has been detected in urine occasionally $(<5 \%$ of infected patients) (7-9).

Because of the rapid spread of SARS-CoV-2, individual screening of clinical cases and study of viral diversity on a population level are challenging. Various reports have demonstrated that enteric and respiratory viruses can be detected in wastewater (10-18). This finding has led to the recognition of wastewaterbased epidemiology as a potentially valuable tool to assess the spread of the disease at a community level. Recently, the Water Research Institute in the Netherlands and other groups have demonstrated temporal correlations between SARS-CoV-2 RNA titers in sewage and the number of reported cases in a city or county when $\geq 26$ gene copies per liter could be detected $(14,19-21)$. Therefore, sewage testing is currently considered globally to be an adjunct to patient-based surveillance and demonstrates promise as an early warning indicator of increasing virus circulation.

${ }^{1}$ These senior authors contributed equally to this article. 
Enhanced surveillance is a key pillar of the current strategy to control the spread of SARS-CoV-2 and includes frequently testing mildly symptomatic persons, investigating infection clusters to identify possible common exposures, and monitoring hospital admission rates. Whole-genome sequencing of SARSCoV-2 from clinical samples has been adopted as an additional tool to identify clusters. Particularly in geographic areas with minimal virus circulation, sequencing can help identify possible sources, provided that sufficient background sequencing has been performed. So far, little work has been done to correlate SARS-CoV-2 diversity in sewage samples with diversity in patients $(22,23)$. We used next-generation sequencing (NGS) of SARS-CoV-2 from wastewater samples to assess whether these samples reflect the diversity of SARS-CoV-2 circulating within the population of the Netherlands and Belgium.

\section{Methods}

\section{Sample Preparation}

Wastewater specimens were collected as 24-h flowdependent composite samples and processed as previously described (14). Debris of 100-200 mL of sewage samples was pelleted and the supernatant was concentrated by using $100 \mathrm{kDa}$ Centricon ultrafilters (Millipore Sigma, https://www.emdmillipore.com); in vitro-transcribed dengue virus type-2 RNA was added as an internal extraction control. RNA was extracted by using the Nuclisens kit (bioMérieux, https:/ /www.biomerieux.com) and KingFisher purification system (Thermo Fisher Scientific, https://www.thermofisher.com) (14). RNA was screened by quantitative reverse transcription PCR (qRT-PCR) with 5 primer-probe sets targeting the SARS-CoV-2 nucleocapsid (N) gene (N1-N3) (24), envelope (E) gene for all sarbecoviruses (25), and the internal control.

\section{NGS}

We performed SARS-CoV-2-specific multiplex PCR for nanopore sequencing as described previously (26). Primers for 89 overlapping amplicons spanning the genome were used in 2 PCR pools. Libraries were generated by using the Oxford Nanopore native barcode kits (Oxford Nanopore Technologies, https:// nanoporetech.com) and sequenced on a R9.4 flow cell.

Illumina sequencing was performed as described previously (27). Amplicons were generated by the multiplex PCR described previously. Amplicons were purified with $0.8 \mathrm{X}$ AMPure XP beads (Beckman Coulter, https://www.beckmancoulter.com) and 100ng of DNA was converted into paired-end Illumina sequencing libraries by using the KAPA HyperPlus library preparation kit (Roche, https:// www.roche.com). We used the KAPA Unique DualIndexed Adapters Kit (Roche) to enable subsequent sequencing of multiple libraries in a single Illumina MiSeq version 3 flowcell $(2 \times 300$ cycles) (Illumina, https:/ / www.illumina.com).

\section{Nanopore Sequence Analysis}

Raw sequence data were processed as previously described (26). We used a snakemake script to demultiplex fastq raw reads by using Porechop (https:/ / github.com/rrwick/Porechop), to trim primers by using Cutadapt (28), and to perform a reference-based alignment by using minimap2 to GISAID sequence EPI_ISL_412973 (https://www.gisaid.org). The run was monitored by using RAMPART (https://articnetwork.github.io/rampart). The consensus genome was extracted by using 2 analyses for which positions with a coverage $<10 \mathrm{X}$ or $<30 \mathrm{X}$ were replaced with an $\mathrm{N}$. We confirmed mutations in the genome by manually checking the alignment in Ugene (29) and resolved homopolymeric regions by consulting reference genomes. On the basis of previous studies (30), we considered mutations with $>30 X$ coverage high quality, whereas mutations $\geq 10 X$ and $\leq 30 X$ coverage were considered low quality.

\section{Illumina Sequence Analysis}

We used a customized Galaxy workflow (31) for all processing, reference-based alignment, and variant analysis. Raw sequencing reads were filtered by using Fastp (32) to remove adaptor contamination, ambiguous bases, low quality reads (Phred score $<30$ ), and fragments $<50$ nt. Reads were mapped against GISAID sequence EPI_ISL_412973 by using the default settings of BWA-MEM (H. Li, unpub. data, https:/ / arxiv.org/abs/1303.3997). Reads were realigned by using the leftalign utility from FreeBayes (E. Garrison, unpub. data, https://arxiv.org/abs/1207.3907). All reads with mapping scores of $<30$ were discarded. Consensus sequences and variants were generated by using iVar (33). Final consensus sequences (frequency $>50 \%$ ) were constructed by using all mapped reads with a coverage of $>5 X$ and Phred score of $>30$. For detection of low-frequency variants (LFVs), we used parameters as follows: minimum coverage of 50X, Phred score $>30$, and a minimum frequency threshold of $10 \%$. Variant calling was confirmed by manual inspection of the aligned reads in Ugene (29). Variant positions are given with respect to the Wuhan-Hu-1 strain (MN908947) (34). We uploaded all consensus 
sequences with coverage $>50 \%$ to GISAID (accession nos. EPI_ISL_539300-25).

\section{Phylogenetic Analysis}

The first dataset included all full-length SARS-CoV-2 genomes from the Netherlands (1,544 genomes) and Belgium (888 genomes) from GISAID as of July 8, 2020. The second dataset was a subsample representative of the global diversity of all SARS-CoV-2 sequences in GISAID as of June 1, 2020. This global dataset contained 2,552 subsampled sequences (full length with Ns $<5 \%$ ) to include 1 unique genome per country or state per week. We aligned sequences with $>75 \%$ genome coverage by using MAFFT (https://mafft.cbrc. jp/alignment/server) and inferred maximum-likelihood trees by using the best predicted models general time-reversible plus F plus R3 (global subsample) and general time-reversible plus F plus R2 (NetherlandsBelgium dataset) and bootstrap with 1,000 replicates. Trees were visualized by using Figtree version 1.4.4 (http://tree.bio.ed.ac.uk/software/figtree). Clades were assigned by using the Nextclade tool.

\section{Results}

\section{Correlation between qRT-PCR and Percentage of Genome Recovered}

Previously, sewage samples collected from 6 locations in the Netherlands (and Schiphol Airport) were tested by qRT-PCR to investigate the levels of SARSCoV-2 RNA (14). To further investigate the genetic diversity of SARS-CoV-2, we subjected 55 wastewater samples obtained from 13 locations in the Netherlands (48 samples) and 7 locations in Belgium (7 samples) with cycle threshold $\left(C_{t}\right)$ values of $<36$ to whole-genome sequencing by using nanopore technology. The wastewater treatment plants in the Netherlands served $\approx 200,000-980,000$ inhabitants; Schiphol was estimated to serve 54,000 persons (14). The samples covered a period of 70 days (March 25-June 3, 2020); of all 55 samples, 2 (Franeker-92719 and AmsterdamWest-92852) were sequenced by nanopore twice. Of the 55 samples, 24 were also sequenced by Illumina (Table 1).

We used 4 primer-probe sets targeting the $\mathrm{N}$ (N1-N3) genes and E gene to evaluate the concentration of SARS-CoV-2 in sewage samples (Table 1) (14). The percentage of the genome covered by the assembly of nanopore reads ( $>10 \mathrm{X}$ coverage) ranged from $0 \%$ to $99.2 \%$. We found an inverse sigmoidal correlation between the percentage of the genome assembled from nanopore sequencing reads and the $\mathrm{N}$ and $\mathrm{E}$ gene $C_{t}$ values (Figure 1). The $C_{t}$ values at which half of the genome could be obtained were 34.6 for N1, 33.8 for N2, 33.2 for N3, and 32.5 for E. No correlation was observed between $C_{t}$ values and the percentage of the genome assembled from Illumina reads (Appendix Figure 1, https:/ / wwwnc.cdc.gov/ EID/article/27/5/20-4410-App1.pdf).

\section{Consensus Sequences}

We performed phylogenetic analysis to assess whether consensus sequences from sewage could be associated with clinical samples from the same region. A total of 22 genomes (20 from nanopore and 2 from Illumina runs) with a coverage $>75 \%$ of the genome were obtained from 20 samples. We used these sequences to infer a maximum-likelihood tree using all sequences from the Netherlands and Belgium available in GISAID and a maximum-likelihood tree using a subset representative of the global diversity of SARS-CoV-2 in GISAID. In general, the sequences from the Netherlands and Belgium grouped into 5 clades (Figure 2, panel A), and most of the sequences belonged to clade 20A (52.0\% for the Netherlands and $47.7 \%$ for Belgium). The clades 19B and 20C were less prevalent; $8.9 \%$ of sequences from the Netherlands belonged to $19 \mathrm{~B}$ and $1.2 \%$ to $20 \mathrm{C}$, whereas $10.4 \%$ of Belgium sequences belonged to $19 \mathrm{~B}$ and $0.3 \%$ to $20 \mathrm{C}$. Both trees showed that sewage samples grouped within clades 19A, 20A, and 20B (Figure 2). Samples Franeker-92719 and HeeswijkDinther-92499 clustered with sequences isolated from patients from the same region (Figure 2, panel $\mathrm{A}$ ), indicating that sewage samples can be linked to specific outbreaks. Included in the phylogenetic trees were 2 samples with 2 consensus sequences (AmsterdamWest-92852 and Franeker-92719), which demonstrated 2-mutation differences between consensus sequences of the same sample (Appendix Table 1). Despite this discrepan$\mathrm{cy}$, consensus sequences from the same sample clustered within the same clade (Appendix Figures 2, 3). Some sequences clustered close to the root of the tree, probably because of the presence of multiple strains within 1 sample, which resulted in a combination of mutations in their consensus sequences.

To associate samples with a particular clade or cluster, we compared all consensus sequences, including partial sequences, with the Wuhan-Hu-1 reference isolate. A total of 145 single-nucleotide polymorphisms (SNPs) were detected in our dataset (Appendix Table 1). Of these, 24 SNPs were detected in $>1$ sequence. We also detected SNPs in the Netherlands sewage sequences with a geographic regional signal, which were present in the Netherlands clinical samples at much higher frequencies than in global or 


\begin{tabular}{|c|c|c|c|c|c|c|c|c|c|c|}
\hline \multirow{2}{*}{$\begin{array}{l}\text { Sample } \\
\text { no. }\end{array}$} & \multirow{2}{*}{$\begin{array}{c}\text { Sample } \\
\text { ID }\end{array}$} & \multirow[b]{2}{*}{ Date } & \multirow[b]{2}{*}{ Country } & \multirow[b]{2}{*}{ Sampling location } & \multicolumn{4}{|c|}{ Target $\mathrm{C}_{\mathrm{t}} \dagger$} & \multicolumn{2}{|c|}{ Coverage, $\%$} \\
\hline & & & & & N1 & N2 & N3 & $\mathrm{E}$ & Nanopore & Illumina \\
\hline 1 & 92499 & 2020 Mar 25 & Netherlands & Heeswijk-Dinther & 32.9 & 32.1 & 30.7 & 30.8 & 94.4 & ND \\
\hline 2 & 92502 & 2020 Mar 25 & Netherlands & Apeldoorn & 36.6 & 34.9 & 33.2 & 33.3 & 74.9 & 19.4 \\
\hline 3 & 92503 & 2020 Mar 25 & Netherlands & Amersfoort & 34.9 & 33.1 & 31.8 & 32.1 & 87.8 & ND \\
\hline 4 & 92504 & 2020 Mar 25 & Netherlands & Utrecht & 31.8 & 30.9 & 29.8 & 29.9 & 95.2 & ND \\
\hline 5 & 92505 & 2020 Mar 25 & Netherlands & Utrecht Overvecht & 32.3 & 31.1 & 30.1 & 30.1 & 92.4 & ND \\
\hline 6 & 92506 & 2020 Mar 25 & Netherlands & Schiphol & 32.7 & 32.0 & 30.8 & 30.7 & 92.2 & 65.6 \\
\hline 7 & 92508 & 2020 Mar 25 & Netherlands & Amsterdam West & 31.8 & 30.7 & 29.7 & 29.9 & 97.0 & ND \\
\hline 8 & 92509 & 2020 Mar 25 & Netherlands & Tilburg & 33.0 & 32.2 & 31.2 & 31.0 & 78.8 & 65.5 \\
\hline 9 & 92719 & 2020 Mar 30 & Netherlands & Franeker & 31.8 & 30.8 & 31.2 & 30.7 & 97.7/50.9‡ & 78.2 \\
\hline 10 & 92721 & 2020 Mar 30 & Netherlands & Beverwijk & 32.6 & 31.4 & 31.8 & 30.8 & 93.7 & 47.7 \\
\hline 11 & 92722 & 2020 Mar 30 & Netherlands & Katwoude & 32.9 & 32.6 & 32.7 & 31.4 & 84.6 & 53.9 \\
\hline 12 & 92723 & 2020 Mar 30 & Netherlands & Wervershoof & 33.1 & 32.3 & 32.5 & 31.1 & 96.6 & 43.2 \\
\hline 13 & 92848 & 2020 Apr 1 & Netherlands & Amersfoort & 33.6 & 32.1 & 32.3 & 31.6 & 96.6 & 39.4 \\
\hline 14 & 92849 & 2020 Apr 1 & Netherlands & Utrecht & 32.4 & 31.4 & 31.8 & 30.6 & 57.8 & 48.5 \\
\hline 15 & 92851 & 2020 Apr 1 & Netherlands & Schiphol & 33.7 & 33.1 & 33.4 & 32.3 & 89.4 & 53.5 \\
\hline 16 & 92852 & 2020 Apr 1 & Netherlands & Amsterdam West & 31.8 & 30.6 & 30.9 & 29.9 & $99.2 / 97.1 \ddagger$ & 59.1 \\
\hline 17 & 92853 & 2020 Apr 1 & Netherlands & Tilburg & 33.5 & 32.6 & 32.6 & 32.0 & 91.2 & ND \\
\hline 18 & 92943 & 2020 Apr 2 & Belgium & Langemark & 33.2 & 33.3 & 33.1 & 32.2 & 60.3 & ND \\
\hline 19 & 92947 & 2020 Apr 2 & Belgium & Lo-Reninge & 34.6 & 34.2 & 34.5 & 33.4 & 71.3 & ND \\
\hline 20 & 92949 & 2020 Apr 2 & Belgium & Properinge & 34.5 & 33.4 & 33.4 & 32.4 & 65.6 & 65.6 \\
\hline 21 & 92965 & 2020 Apr 2 & Netherlands & Delft & 32.9 & 32.9 & 32.9 & 31.5 & 91.7 & 52.4 \\
\hline 22 & 93030 & 2020 Apr 5 & Belgium & Aartselaar & 33.2 & 32.4 & 31.6 & 31.4 & 89.9 & 61.2 \\
\hline 23 & 93032 & 2020 Apr 5 & Belgium & Gent & 34.2 & 33.7 & 32.6 & 32.1 & 63.2 & 46.9 \\
\hline 24 & 93034 & 2020 Apr 5 & Belgium & Leuven & 33.6 & 33.4 & 32.1 & 31.4 & 70.2 & 37.6 \\
\hline 25 & 93036 & 2020 Apr 5 & Belgium & Tienen & 33.3 & 32.6 & 31.2 & 30.8 & 88.1 & 41.5 \\
\hline 26 & 93818 & $2020 \mathrm{Apr} 8$ & Netherlands & Amersfoort & 34.9 & 34.3 & 33.4 & 32.4 & 37.5 & ND \\
\hline 27 & 93820 & $2020 \mathrm{Apr} 9$ & Netherlands & Utrecht & 32.8 & 32.2 & 31.2 & 30.8 & 55.2 & ND \\
\hline 28 & 93822 & 2020 Apr 9 & Netherlands & Amsterdam West & 32.6 & 25.1 & 31.6 & 30.9 & 87.3 & ND \\
\hline 29 & 93823 & 2020 Apr 9 & Netherlands & Schiphol & 33.0 & 33.2 & 32.2 & 31.3 & 67.3 & 43.5 \\
\hline 30 & 93825 & 2020 Apr 8 & Netherlands & Delft & 33.9 & 33.7 & 32.7 & 32.0 & 63.9 & 64.3 \\
\hline 31 & 93828 & 2020 Apr 9 & Netherlands & Tilburg & 35.2 & 34.6 & 33.1 & 32.7 & 31.2 & ND \\
\hline 32 & 93948 & 2020 Apr 14 & Netherlands & Heeswijk-Dinther & 35.8 & 34.6 & 33.6 & 32.7 & 18.8 & ND \\
\hline 33 & 93950 & 2020 Apr 15 & Netherlands & Wervershoof & 34.9 & 34.3 & 33.1 & 32.5 & 60.7 & ND \\
\hline 34 & 94330 & 2020 Apr 21 & Netherlands & Utrecht1 & 35.1 & 34.4 & 33.2 & 33.5 & 41.7 & ND \\
\hline 35 & 94331 & 2020 Apr 21 & Netherlands & Utrecht2 & 35.7 & 34.1 & 34.2 & 33.7 & 38.6 & ND \\
\hline 36 & 94334 & 2020 Apr 21 & Netherlands & Amsterdam West & 34.0 & 33.3 & 32.4 & 32.0 & 66.7 & ND \\
\hline 37 & 94335 & 2020 Apr 21 & Netherlands & Schiphol & 33.8 & 34.1 & 32.9 & 33.7 & 40.1 & 43.0 \\
\hline 38 & 94337 & 2020 Apr 21 & Netherlands & Delft & 35.7 & 34.1 & 34.1 & 33.8 & 34.2 & ND \\
\hline 39 & 94339 & 2020 Apr 21 & Netherlands & Tilburg & 34.8 & 35.4 & 34.7 & 36.0 & 11.2 & 80.3 \\
\hline 40 & 94602 & 2020 Apr 29 & Netherlands & Utrecht & 35.6 & 34.3 & 33.0 & 34.2 & 29.6 & ND \\
\hline 41 & 94604 & 2020 Apr 29 & Netherlands & Amsterdam West & 34.9 & 34.6 & 32.8 & 33.6 & 15.0 & ND \\
\hline 42 & 94605 & 2020 Apr 29 & Netherlands & Schiphol & 34.6 & 35.1 & 33.6 & 33.2 & 21.3 & 35.2 \\
\hline 43 & 94607 & 2020 Apr 25 & Netherlands & Delft & 35.8 & 36.2 & 34.4 & 34.0 & 15.3 & ND \\
\hline 44 & 94976 & 2020 May 7 & Netherlands & Utrecht & 35.5 & 36.0 & 35.1 & 33.5 & 6.3 & ND \\
\hline 45 & 94978 & 2020 May 7 & Netherlands & Amsterdam West & 35.0 & 34.8 & 34.5 & 33.7 & 19.8 & ND \\
\hline 46 & 94982 & 2020 May 6 & Netherlands & Delft & 35.1 & 35.9 & 34.7 & 33.7 & 18.7 & ND \\
\hline 47 & 95550 & 2020 May 13 & Netherlands & Utrecht & ND & 34.4 & ND & 32.0 & 3.0 & ND \\
\hline 48 & 95552 & 2020 May 13 & Netherlands & Amsterdam West & ND & 34.2 & ND & 32.8 & 20.4 & ND \\
\hline 49 & 95556 & 2020 May 12 & Netherlands & Delft & ND & 34.4 & ND & 34.1 & 0 & ND \\
\hline 50 & 95558 & 2020 May 13 & Netherlands & Tilburg & ND & 34.3 & ND & 36.1 & 0 & ND \\
\hline 51 & 95793 & 2020 May 19 & Netherlands & Utrecht & ND & 35.1 & ND & 34.9 & 0 & ND \\
\hline 52 & 95794 & 2020 May 19 & Netherlands & Amsterdam West & ND & 35.1 & ND & 34.2 & 7.7 & ND \\
\hline 53 & 96925 & 2020 Jun 2 & Netherlands & Utrecht & ND & 35.2 & ND & 37.1 & 0 & ND \\
\hline 54 & 96927 & 2020 Jun 2 & Netherlands & Schiphol & ND & 32.5 & ND & 31.1 & 30.8 & 34.0 \\
\hline 55 & 97044 & 2020 Jun 3 & Netherlands & Delft & ND & 35.7 & ND & 33.5 & 8.2 & ND \\
\hline
\end{tabular}

${ }^{*} \mathrm{C}_{\mathrm{t}}$, cycle threshold; $\mathrm{E}$, envelope; $\mathrm{N}$, nucleocapsid; ND, not determined; SARS-CoV-2, severe acute respiratory syndrome coronavirus 2. †Three primer-probe sets targeting the N1-N3 genes and 1 targeting the E gene.

$\ddagger$ These samples were sequenced twice. 


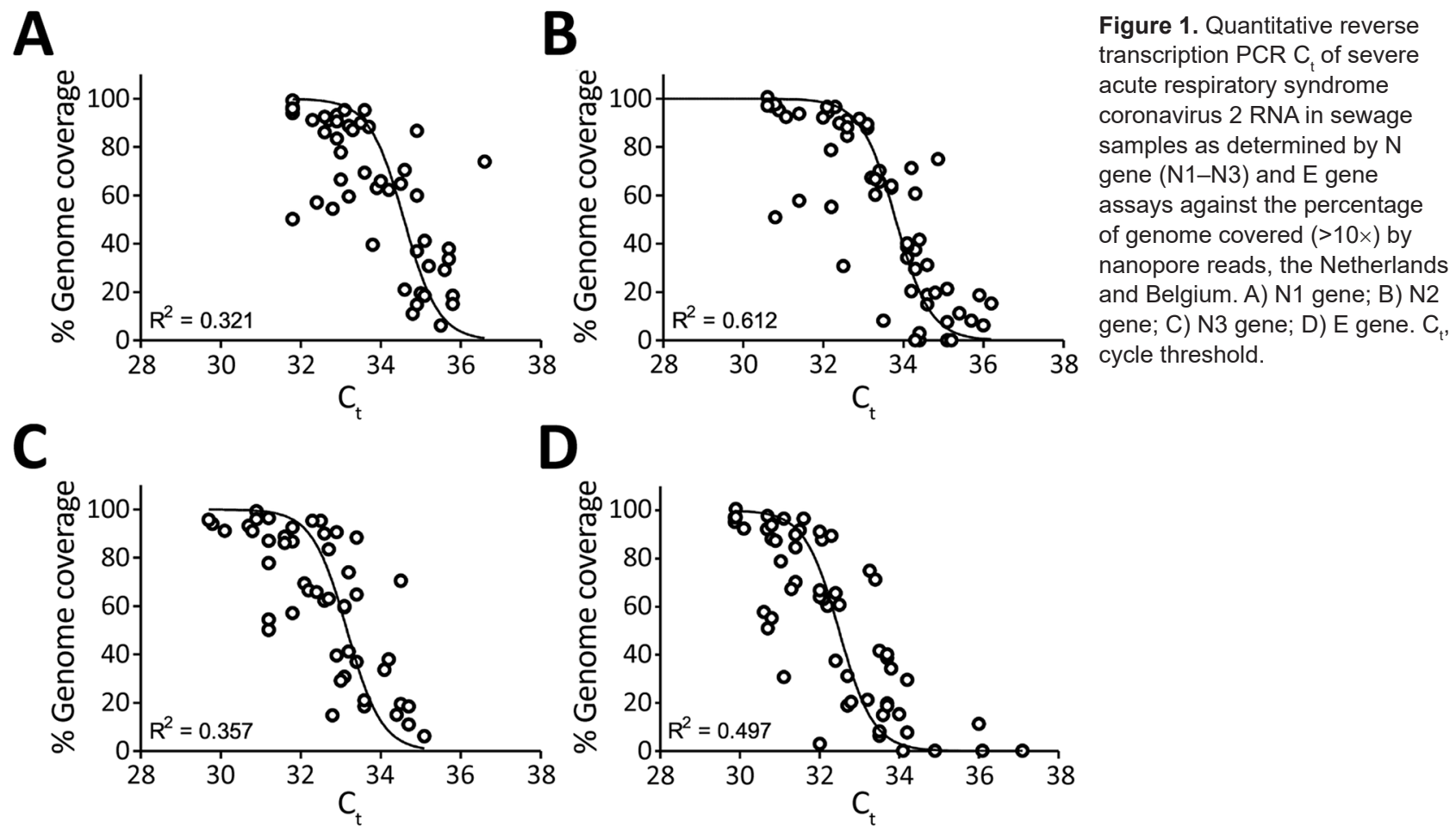

clade within a sample; the presence of these mutations can also aid in the detection of virus mixtures in a sample. During the period of wastewater-sample collection, Nextstrain defined 5 major clades (19A, 19B, 20A, 20B, and 20C). Each clade is defined by the presence of $\geq 2$ linked mutations. Clade $19 \mathrm{~A}$ is the root clade and contains the Wuhan-Hu-1 reference sequence. Both 19B and 20A emerged from 19A, where 2 and 3 linked mutations define these major clades: T28144C and C8782T define 19B; and C3037T, C14408T, and A23403G define 20A. Clades 20B and 20C emerged from 20A, where the trinucleotide substitution GGG28881-28883AAC defines 20B and the linked mutations C1059T and G25563T define 20C. Nucleotide substitution A23403G, a signature mutation of clades 20A, 20B, and 20C that generates the D614G amino-acid substitution in the $S$ glycoprotein, was detected in $83.6 \%(51 / 61)$ of the samples that were sequenced at this region (Appendix Table 1). The GGG28881-28883AAC substitution was detected in $41.9 \%(18 / 43)$ of the sequences. One of the 2 mutations defining the low-prevalence clades 20C and 19B (C1059T and T28144C) was found in 2 and 3 consensus sequences. However, these sequences could not be assigned to these clades because regions containing the additional clade-defining mutations were not sequenced with sufficient coverage. The hCoV-19/env/ Netherlands/ Amersfoort-92503-N/2020 sequence contained a mix of clade-defining mutations: C1059T, which defines 20C; T28144C, which defines 19B; and GGG28881-28883AAC, which defines 20B. This finding indicates that the obtained consensus sequence does not represent a single strain.

In addition to the clade-defining mutations, we detected 49 and 63 SNPs that were not present in either the Netherlands (1,544 sequences) or Belgium (888 sequences) datasets but were seen in the global dataset (55,074 sequences), although with $<1 \%$ prevalence (Appendix Table 2). Moreover, we detected 51 novel mutations in sewage consensus sequences that were not previously reported, of which 48 were supported by coverage above the thresholds set for high quality (coverage $>30 \times$ for Nanopore and coverage $>5 x$ and Phred score $>30$ for Illumina). Discrepancies between consensus sequences of the same sewage sample can occur. AmsterdamWest-92852 was sequenced 3 times and 4 positions varied (Appendix Table 1). These differences are explained by the presence of variant sites in a single sample in similar percentages, which resulted in differences in consensus sequences between sequencing runs.

\section{LFV Analysis}

Given that sewage samples are likely to contain a mixture of SARS-CoV-2 strains, we performed a variant analysis with Illumina data to distinguish multiple strains within single samples. By using a coverage $>50 \times$, Phred score $>30$, and a frequency threshold of 
$>10 \%$ as settings, we found 21 positions with at least 1 sample containing major and minor variants (Table 2). Of these, 14 mutations resulted in changes at the amino acid level (12 nonsynonymous mutations and 2 deletions). Of note, 8 of these (4497C, 10514C, 11484T, 13046A， 16538_16540delATA， 16777T, 16823T, and 28736A) are novel mutations that did not appear in the Netherlands-Belgium or global datasets. The other 7 variants appeared but demonstrated low prevalence in both datasets $(0.002 \%-0.130 \%)$. The most prominent of these was the 28139A mutation in a wastewater sample from March, which was detected in only 4 sequences worldwide and demonstrated both a strong temporal (all detected in March 2020)

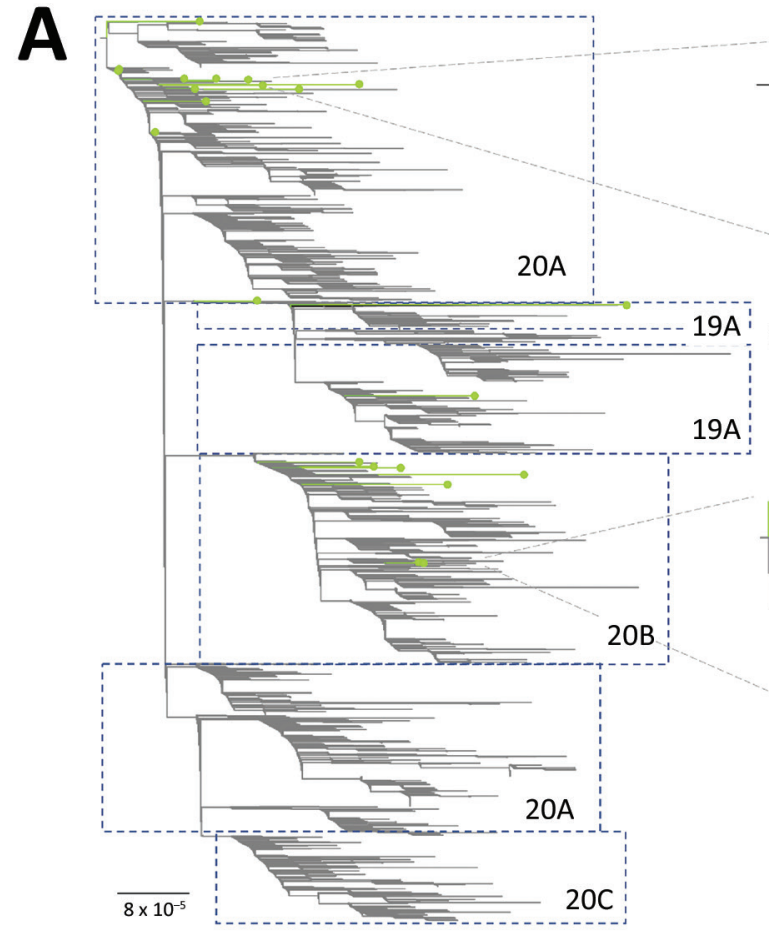

B

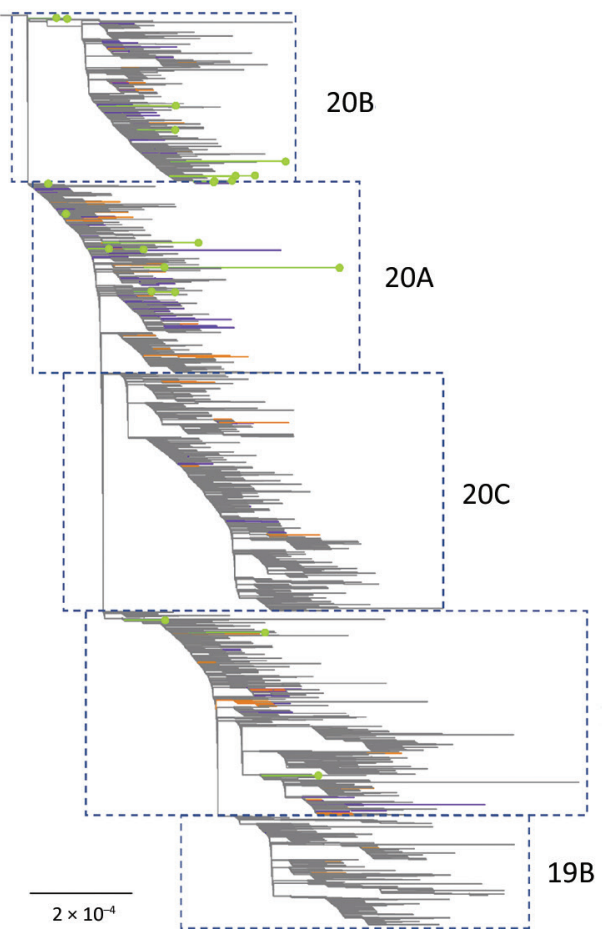

hCoV-19_Netherlands_NoordBrabant_137_2020_EPI_ISL_461172_2020-04-15_Europe hCoV-19_Netherlands_Zeeland_16_2020_EPI_ISL_461254_2020-03-28_Europe - hCoV-19_Netherlands_NA_241_2020_EPI_ISL_422775_2020-03-27_Europe - hCoV-19_Netherlands_NA_439_2020_EPI_ISL_455241_2020-04-09_Europe hCov-19/env/Netherlands/HeeswijkDinther-92499-N/2020 hCoV-19/env/Netherlands/Schiphol-92506-N/2020 $2 \times 10^{-5}$

$\longrightarrow \mathrm{hCoV}-19 / \mathrm{env} /$ Netherlands/Franeker-92719-N1/2020 - hCov-19/env/Netherlands/Franeker-92719-1/2020 hCoV-19_Netherlands_Friesland_43_2020_EPI_ISL_460858_2020-05-04_Europe - hCoV-19_Netherlands_Friesland_38_2020_EPI_ISL_460853_2020-04-14_Europe hCoV-19_Netherlands_NA_588_2020_EPI_ISL_460752_2020-00-00_Europe hCoV-19_Netherlands_Friesland_7_2020_EPI_ISL_422572_2020-03-28_Europe hCoV-19_Netherlands_NA_587_2020_EPI_ISL_460751_2020-00-00_Europe $\overline{2 \times 10^{-5}}$

Figure 2. Phylogenetic analysis of severe acute respiratory syndrome coronavirus 2 genome consensus sequences detected in sewage samples, the Netherlands and Belgium. A) The Netherlands subsample dataset; B) global subsample dataset. Lines with dots in green indicate samples sequenced in this study. Clades (19A, 19B, 20A, 20B, and 20C) were assigned by using the Nextclade tool (https://clades.nextstrain.org). For the global subsample tree, samples in orange indicate the Netherlands sequences. Samples in purple indicate Belgium sequences. Scale bars indicate inferred number of nucleotide substitutions per site. 
Table 2. Summary of LFVs detected in wastewater samples determined by Illumina sequencing in study of SARS-CoV-2 circulation and diversity through community wastewater sequencing, the Netherlands and Belgium*

\begin{tabular}{|c|c|c|c|c|c|c|c|c|c|c|c|}
\hline \multirow[b]{2}{*}{ Positiont } & \multirow[b]{2}{*}{ Sample } & \multirow[b]{2}{*}{ MV } & \multirow[b]{2}{*}{ LFV } & \multirow[b]{2}{*}{ LFV, \% } & \multirow{2}{*}{$\begin{array}{l}\text { Total } \\
\text { depth }\end{array}$} & \multirow[b]{2}{*}{ Feature } & \multirow[b]{2}{*}{ AA MV } & \multirow{2}{*}{$\begin{array}{l}\text { AA } \\
\text { LFV }\end{array}$} & \multicolumn{3}{|c|}{ Frequency, \% } \\
\hline & & & & & & & & & $\mathrm{NL}$ & $\mathrm{BE}$ & Global \\
\hline 1440 & NL/Schiphol-92506-I & $\mathrm{G}$ & A & 13.2 & 53 & ORF1a & $\mathrm{G}$ & $\mathrm{N}$ & 1.619 & 4.167 & 1.903 \\
\hline 3549 & NL/Franeker-92719-I & $\begin{array}{l}\text { GACCA } \\
\text { CTTA }\end{array}$ & $-\S$ & 46.8 & 201 & ORF1a & GPLK & $E$ & 0 & 0 & 0 \\
\hline 4497 & NL/Beverwijk-92721-I & $\mathrm{T}$ & C & 42.6 & 479 & ORF1a & I & $\mathrm{T}$ & 0 & 0 & 0.000 \\
\hline 10514 & $\begin{array}{c}\text { NL/AmsterdamWest- } \\
\text { 92852-I }\end{array}$ & $\mathrm{T}$ & C & 12.5 & 1,656 & ORF1a & $\mathrm{Y}$ & $\mathrm{H}$ & 0 & 0 & 0 \\
\hline \multirow[t]{2}{*}{10933} & BE/Aartselaar-93030-I & C & $T$ & 18.0 & 50 & ORF1a & $\mathrm{P}$ & $\mathrm{P}$ & 100.000 & 100.000 & 99.996 \\
\hline & NL/Tilburg-94339-I & $\mathrm{T}$ & C & 11.1 & 63 & ORF1a & $P$ & $P$ & 0 & 0 & 0.004 \\
\hline \multirow[t]{3}{*}{11083} & BE/Properinge-92949-I & $\mathrm{G}$ & $\mathrm{T}$ & 12.1 & 58 & ORF1a & $\mathrm{L}$ & $\mathrm{F}$ & 5.635 & 7.320 & 11.007 \\
\hline & BE/Aartselaar-93030-I & $\mathrm{T}$ & G & 26.4 & 129 & ORF1a & $\mathrm{F}$ & L & 94.430 & 92.680 & 88.069 \\
\hline & NL/Tilburg-94339-I & G & $\mathrm{T}$ & 12.7 & 150 & ORF1a & $\mathrm{L}$ & $\mathrm{F}$ & 5.635 & 7.320 & 11.007 \\
\hline \multirow[t]{2}{*}{11109} & $\begin{array}{c}\text { NL/AmsterdamWest- } \\
\text { 92852-I }\end{array}$ & C & $\mathrm{T}$ & 48.3 & 230 & ORF1a & A & V & 15.220 & 0.338 & 0.534 \\
\hline & NL/Tilburg-94339-I & C & T & 21.2 & 66 & ORF1a & A & V & 15.220 & 0.338 & 0.534 \\
\hline 11484 & NL/Beverwijk-92721-I & $\mathrm{C}$ & $T$ & 44.0 & 84 & ORF1a & A & V & 0 & 0 & 0 \\
\hline \multirow[t]{3}{*}{11494} & NL/Franeker-92719-I & C & $T$ & 13.5 & 104 & ORF1a & $\mathrm{N}$ & $\mathrm{N}$ & 0 & 0 & 0.002 \\
\hline & BEAartselaar-93030-I & $\mathrm{C}$ & $\mathrm{T}$ & 43.5 & 370 & ORF1a & $\mathrm{N}$ & $\mathrm{N}$ & 0 & 0 & 0.002 \\
\hline & NL/Tilburg-94339-I & $\mathrm{C}$ & $\mathrm{T}$ & 13.8 & 247 & ORF1a & $\mathrm{N}$ & $\mathrm{N}$ & 0 & 0 & 0.002 \\
\hline 13046 & BE/Aartselaar-93030-I & $\mathrm{C}$ & A & 36.7 & 98 & ORF1a & $\mathrm{P}$ & $\mathrm{T}$ & 0 & 0 & 0 \\
\hline 13426 & BE/Gent-93032-I & C & $\mathrm{T}$ & 22.6 & 115 & ORF1a & $\mathrm{R}$ & $\mathrm{R}$ & 0 & 0 & 0.038 \\
\hline 16538 & BE/Gent-93032-I & - & ATA & 27.6 & 348 & ORF1b & - & $\mathrm{N}$ & 100.000 & 100.000 & 100.000 \\
\hline 16777 & NL/Schiphol-92851-I & G & $\mathrm{T}$ & 30.2 & 404 & ORF1b & V & $\mathrm{F}$ & 0 & 0 & 0 \\
\hline 16806 & NL/Tilburg-94339-I & C & $A$ & 22.1 & 77 & ORF1b & $\mathrm{N}$ & K & 0 & 0 & 0.016 \\
\hline 16823 & BE/Aartselaar-93030-I & G & $\mathrm{T}$ & 12.0 & 192 & ORF1b & G & V & 0 & 0 & 0 \\
\hline 24862 & NL/Katwoude-92722-I & A & G & 34.0 & 53 & S & $\mathrm{T}$ & $\mathrm{T}$ & 8.614 & 0.338 & 0.463 \\
\hline 28115 & NL/Delft-92965-I & $\mathrm{T}$ & C & 47.8 & 67 & ORF8 & i & i & 100.000 & 100.000 & 99.993 \\
\hline 28139 & NL/Tilburg-94339-I & $\mathrm{C}$ & $A$ & 36.0 & 136 & ORF8 & $\mathrm{S}$ & $\mathrm{S}$ & 0.130 & 0.113 & 0.007 \\
\hline 28375 & NL/Tilburg-94339-I & G & A & 30.8 & 146 & $\mathrm{~N}$ & G & G & 0 & 0 & 0.002 \\
\hline \multirow[t]{3}{*}{28394} & $\begin{array}{c}\text { NL/AmsterdamWest- } \\
92852-I\end{array}$ & $\mathrm{C}$ & $\mathrm{T}$ & 31.5 & 54 & $\mathrm{~N}$ & $\mathrm{R}$ & W & 0 & 0 & 0.004 \\
\hline & BE/Properinge-92949-I & C & $\mathrm{T}$ & 16.7 & 60 & $\mathrm{~N}$ & $\mathrm{R}$ & W & 0 & 0 & 0.004 \\
\hline & NL/Tilburg-94339-I & C & $\mathrm{T}$ & 13.6 & 191 & $\mathrm{~N}$ & $\mathrm{R}$ & W & 0 & 0 & 0.004 \\
\hline 28736 & BE/Leuven-93034-I & A & G & 22.0 & 363 & $\mathrm{~N}$ & A & $\mathrm{T}$ & 100.000 & 100.000 & 100.000 \\
\hline
\end{tabular}

*BE, Belgium; LFV, low-frequency variant; MV, major variant; NL, Netherlands; SARS-CoV-2, severe acute respiratory syndrome coronavirus 2. †Positions are given with respect to Wuhan-Hu-1 (GenBank accession no. MN908947).

†Frequency of the LFV of sample against GISAID database (as of July 8, 2020) of the Netherlands, Belgium, and global samples.

§Dashes represent a gap at the given region, either as a MV or LFV.

and regional signal (2 sequences from the Netherlands [EPI_ISL_422640 and EPI_ISL_422880], 1 from Denmark [EPI_ISL_444879], and 1 from Belgium [EPI_ISL_458209]).

Finally, 4 variants $(1440 \mathrm{~A}, 11083 \mathrm{~T}, 11109 \mathrm{~T}$, and $24862 \mathrm{G})$ appeared at higher levels in both datasets $(>0.5 \%) ; 11109 \mathrm{~T}$ and $24862 \mathrm{G}$ were 28.5 and 14.3 times more prevalent in the Netherlands dataset than in the global dataset (Table 2). The other variants appeared at similar frequencies in all datasets.

In addition to consensus sequences, LFV analysis is of value for identifying potential local outbreaks. This identification could be achieved by detecting cluster-defining mutations that are associated with sequences from a particular geographic area. To associate the presence of a minor variant to sequences belonging to unique clusters, we mapped the 4 most prevalent LFVs onto the Netherlands-Belgium subsample and global subsample phylogenetic trees (Figure 3). For 3 variants (1440A, 11109T, and 24862G), the presence of the mutation and their clustering on the phylogenies were clearly associated. However, when 1 of these 3 variants was detected as an LFV in a sewage sample, the consensus sequence of this sample did not group with the cluster of clinical samples that contains the variant. For example, the $24862 \mathrm{G}$ variant in sample Tilburg-94339 was detected in 2 unique clusters within clade 20A, whereas its consensus sequence (hCoV-19/env/Netherlands/Tilburg94339-I/2020) clustered within clade 20B, suggesting the presence of both clades in this sample. Although mutation 11083T was most prevalent in clade 19A, it was also scattered along the trees, indicating poor association with a particular clade.

\section{Discussion}

The use of wastewater sampling as a tool to learn more about the epidemiology and diversity of SARS-CoV-2 at a community level offers many advantages over human sampling. Sewage samples are relatively easy to 
collect, sampling bias toward severe cases does not occur, ethical issues are limited, and potentially fewer samples are required to determine temporal changes of viral infections in the community $(35,36)$. Nevertheless, comprehensive comparisons with clinical surveillance are required to determine the extent and limits of using sewage as a surveillance or early-warning tool.

We used nanopore and Illumina NGS analysis to study the diversity of SARS-CoV-2 in sewage and compared these results to the viral diversity found in clinical samples. To evaluate this diversity in a comprehensive fashion, we used the Nextstrain clade classification system because it is based on the use of signature mutations to assign sequences to a clade (3), enabling the association of SNPs or LFV to a particular clade, especially for genome sequences with $<75 \%$ coverage.

Our method enabled us to obtain complete or nearcomplete genomes from wastewater samples with $C_{t}$ values of $\geq 5 \mathrm{C}_{\mathrm{t}} \mathrm{s}$ below the limit of detection and partial genomes for samples with higher $C_{t}$ values. To increase the percentage of genome covered, a threshold of $10 \times$ coverage per position was used to generate consensus sequences from nanopore reads. The error rate with this threshold is $<0.03 \%$, and most of the mutations $(132 / 145)$ listed have a coverage of $>30 \times$, which produces an error rate of $1 / 585,000 \mathrm{nt}(30)$.

Of note, we found sewage samples that clustered with sequences isolated from patients of the same region and LFV with a strong regional signal. In a recent study from the United States, wastewater contained SARS-CoV-2 genomes identical to those in clinical samples from the same region (37). Sewage samples can contain a mixture of SARS-CoV-2 viruses, which can be an indication of multiple viruses circulating within a community and perhaps in domestic and livestock animals (38-42). We applied a targeted amplification method and thus did not assess the presence of other viruses. Consensus sequence genomes from a wastewater sample can identify the predominant virus strain in a population, which is suitable for locations with few introductions of the virus $(22,23)$. However, this approach is not appropriate for a population in which multiple virus strains are circulating in parallel. Moreover, it might lead to artificial consensus genomes that do not represent an existing virus.

NGS analysis can unravel the diversity of viruses within a complex sample such as wastewater, particularly by using unbiased sequencing of the sewage virome (43). Nevertheless, the detection of variants of a virus in a single sample can be challenging because of the relatively low number of reads obtained for each virus. Targeted amplification and NGS of a small genome region of the virus of interest to determine the prevalence of virus variants within a single wastewater sample is more sensitive and less expensive; use of this approach has been reported for enteroviruses, human mastadenoviruses, and noroviruses $(12,18,44)$. Because the diversity of SARS-CoV-2 is still limited,

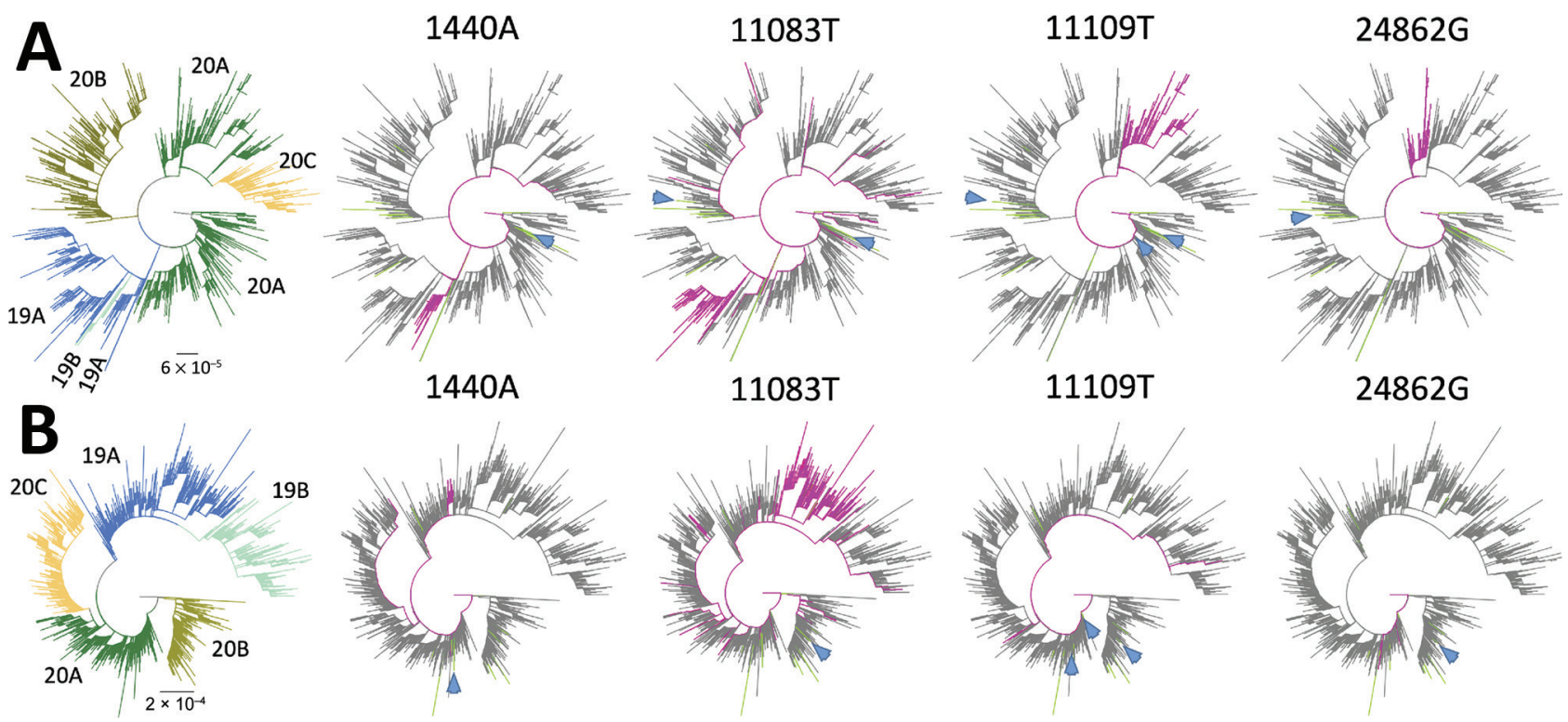

Figure 3. Phylogenetic trees showing 4 low-frequency variants detected in sewage samples in study of severe acute respiratory syndrome coronavirus 2 circulation and diversity through community wastewater sequencing, the Netherlands and Belgium. A) The Netherlands-Belgium subsample; B) global subsample. Patient sequences containing the mutation are shown in magenta. Lines in green indicate sewage samples sequenced in this study. Clades (19A, 19B, 20A, 20B, and 20C) are indicated in colors at the left of the figure. Blue arrows show the consensus sequences (if available) of the sewage samples in which the low-frequency variant was detected. Scale bars indicate the inferred number of nucleotide substitutions per site. 
however, this approach would not be useful since no single small piece of the genome can reliably differentiate between clades or lineages. However, we demonstrated that some LFVs and SNPs can be linked to particular clusters or clades within trees without the need for a complete genome. To confidently determine the presence of a particular cluster within a sample, at least 2 LFVs associated with the cluster should be present at substantial levels. Furthermore, variant analysis can also be used to monitor the prevalence of biologically relevant mutations, such as D614G, which has been shown to increase infectivity in vitro (45) and might be associated with higher transmission and death rates (46; M. Cortey, unpub. data, https://www.biorxiv. org/content/10.1101/2020.05.16.099499v1). Within our dataset, clear temporal changes in the prevalence of LFVs or SNPs in sewage samples that correlated with changes in the clinical dataset were not detected during the first wave.

The combination of whole-genome sequencing of clinical samples with epidemiologic data is vital for public health decision-making (26) because it helps identify clusters of infection, new introductions of virus, and the expansion and decline of circulating strains. Cities with large numbers of visitors are expected to experience several introductions of the virus, whereas the opposite is expected for cities with low numbers of visitors. The use of NGS analysis of sewage samples to evaluate viral diversity within a geographic area and its changes over time can aid in decision-making. For example, in scenarios in which a large increase of viral diversity is detected in sewage, suggesting new introductions of virus, appropriate measures can be taken.

Wastewater can also be used to monitor novel mutations. Our consensus and LFV analyses revealed 57 mutations that were not seen in the global database. These novel mutations might not have been detected for several reasons: they represent technical errors; the mutations did not stay within the population; or the mutations are associated with asymptomatic or mild disease, viruses from animal hosts, enteric shedding, or defective genomes. The presence of defective genomes has previously been suggested for the detection of LFVs that generate stop codons in clinical samples (47). Phenotypic studies could help determine the likelihood and biologic relevance of these novel mutations.

In conclusion, this study illustrates the value of NGS analysis of wastewater to approximate the diversity of SARS-CoV-2 circulating in a community. Sequencing of wastewater samples could be a powerful tool to complement clinical surveillance or could be used independently in settings in which wide clinical sequencing is unfeasible. In addition, in-depth NGS analysis of wastewater samples can help in assessing changes in viral diversity, which can indicate the emergence of epidemiologically or clinically relevant mutations and thereby aid public health decision-making.

This article was preprinted at https:/ / www.medrxiv.org/ content/10.1101/2020.09.21.20198838v1.

\section{Acknowledgments}

We thank the Water Authorities in the Netherlands (Aa en Maas, Amstel Gooi en Vecht, Delfland, De Dommel, Fryslan, Hollands Noorderkwartier, Stichtse Rijnlanden, Vallei en Veluwe, Evides, Waternet) and Belgium (Aquafin, De Watergroep) for the provision of the sewage samples. We thank Pelle van der Wal for his help with the Illumina MiSeq runs. We gratefully acknowledge the authors originating and submitting laboratories of the global sequences from the GISAID EpiCoV Database (1), on which this research is based.

This work was supported by the European Union's Horizon H2020 grants VEO (grant no. 874735) and METASTAVA (grant no. 773830), the Erasmus MC foundation, and the Adessium Foundation.

\section{About the Author}

Mr. Izquierdo-Lara is a PhD student in the Department of Viroscience, Erasmus University Medical Center, Rotterdam, the Netherlands. His research interests are chronic norovirus infections in immunocompromised patients and virus evolution and emergence.

\section{References}

1. Shu Y, McCauley J. GISAID: Global initiative on sharing all influenza data - from vision to reality. Euro Surveill. 2017;22:30494. https://doi.org/10.2807/1560-7917. ES.2017.22.13.30494

2. Rambaut A, Holmes EC, O'Toole Á, Hill V, McCrone JT, Ruis C, et al. A dynamic nomenclature proposal for SARS$\mathrm{CoV}-2$ lineages to assist genomic epidemiology. Nat Microbiol. 2020;5:1403-7. https:/ / doi.org/10.1038/s41564-020-0770-5

3. Hadfield J, Megill C, Bell SM, Huddleston J, Potter B, Callender C, et al. Nextstrain: real-time tracking of pathogen evolution. Bioinformatics. 2018;34:4121-3. https:// doi.org/10.1093/bioinformatics/bty407

4. Lamers MM, Beumer J, van der Vaart J, Knoops K, Puschhof J, Breugem TI, et al. SARS-CoV-2 productively infects human gut enterocytes. Science. 2020;369:50-4. https://doi.org/10.1126/science.abc1669

5. Xiao F, Tang M, Zheng X, Liu Y, Li X, Shan H. Evidence for gastrointestinal infection of SARS-CoV-2. Gastroenterology. 2020;158:1831-1833.e3. https:/ / doi.org/10.1053/ j.gastro.2020.02.055 
6. Xiao F, Sun J, Xu Y, Li F, Huang X, Li H, et al. Infectious SARS-CoV-2 in feces of patient with severe COVID-19. Emerg Infect Dis. 2020;26:1920-2. https://doi.org/10.3201/ eid2608.200681

7. Parasa S, Desai M, Thoguluva Chandrasekar V, Patel HK, Kennedy KF, Roesch T, et al. Prevalence of gastrointestinal symptoms and fecal viral shedding in patients with coronavirus disease 2019: a systematic review and meta-analysis. JAMA Netw Open. 2020;3:e2011335. https:// doi.org/10.1001/jamanetworkopen.2020.11335

8. Jones DL, Baluja MQ, Graham DW, Corbishley A, McDonald JE, Malham SK, et al. Shedding of SARS-CoV-2 in feces and urine and its potential role in person-to-person transmission and the environment-based spread of COVID-19. Sci Total Environ. 2020;749:141364. https://doi.org/10.1016/j.scitotenv.2020.141364

9. Foladori P, Cutrupi F, Segata N, Manara S, Pinto F, Malpei F, et al. SARS-CoV-2 from faeces to wastewater treatment: what do we know? A review. Sci Total Environ. 2020;743:140444. https:/ /doi.org/10.1016/j.scitotenv.2020.140444

10. Strubbia S, Phan MVT, Schaeffer J, Koopmans M, Cotten M, Le Guyader FS. Characterization of norovirus and other human enteric viruses in sewage and stool samples through next-generation sequencing. Food Environ Virol. 2019;11:400-9. https:/ / doi.org/10.1007/s12560-019-09402-3

11. Hellmér M, Paxéus N, Magnius L, Enache L, Arnholm B, Johansson A, et al. Detection of pathogenic viruses in sewage provided early warnings of hepatitis A virus and norovirus outbreaks. Appl Environ Microbiol. 2014;80:6771-81. https:/ / doi.org/10.1128/AEM.01981-14

12. Bisseux M, Colombet J, Mirand A, Roque-Afonso A-M, Abravanel F, Izopet J, et al. Monitoring human enteric viruses in wastewater and relevance to infections encountered in the clinical setting: a one-year experiment in central France, 2014 to 2015. Euro Surveill. 2018;23:17-00237. https:/ / doi.org/10.2807/1560-7917.ES.2018.23.7.17-00237

13. Wang $W, X u Y$, Gao R, Lu R, Han K, Wu G, et al. Detection of SARS-CoV-2 in different types of clinical specimens. JAMA. 2020;323:1843-4. https://doi.org/10.1001/jama.2020.3786

14. Medema G, Heijnen L, Elsinga G, Italiaander R, Brouwer A. Presence of SARS-coronavirus-2 RNA in sewage and correlation with reported COVID-19 prevalence in the early stage of the epidemic in the Netherlands. Environ Sci Technol Lett. 2020;7:511-6. https://doi.org/10.1021/ acs.estlett.0c00357

15. Heijnen L, Medema G. Surveillance of influenza A and the pandemic influenza A (H1N1) 2009 in sewage and surface water in the Netherlands. J Water Health. 2011;9:434-42. https:/ / doi.org/10.2166/wh.2011.019

16. Patel JC, Diop OM, Gardner T, Chavan S, Jorba J, Wassilak SGF, et al. Surveillance to track progress toward polio eradicationworldwide, 2017-2018. MMWR Morb Mortal Wkly Rep. 2019;68:312-8. https://doi.org/10.15585/mmwr.mm6813a4

17. Suffredini E, Iaconelli M, Equestre M, Valdazo-González B, Ciccaglione AR, Marcantonio C, et al. Genetic diversity among genogroup II noroviruses and progressive emergence of GII.17 in wastewaters in Italy (2011-2016) revealed by next-generation and Sanger sequencing. Food Environ Virol. 2018;10:141-50. https://doi.org/10.1007/s12560-017-9328-y

18. Fumian TM, Fioretti JM, Lun JH, Dos Santos IAL, White PA, Miagostovich MP. Detection of norovirus epidemic genotypes in raw sewage using next generation sequencing. Environ Int. 2019;123:282-91. https://doi.org/ 10.1016/j.envint.2018.11.054

19. Wu F, Zhang J, Xiao A, Gu X, Lee WL, Armas F, et al. SARS-CoV-2 titers in wastewater are higher than expected from clinically confirmed cases. mSystems. 2020;5:e00614-20. https://doi.org/10.1128/mSystems.00614-20

20. Randazzo W, Truchado P, Cuevas-Ferrando E, Simón P, Allende A, Sánchez G. SARS-CoV-2 RNA in wastewater anticipated COVID-19 occurrence in a low prevalence area. Water Res. 2020;181:115942. https:/ / doi.org/10.1016/ j.watres.2020.115942

21. Wurtzer S, Marechal V, Mouchel JM, Maday Y, Teyssou R, Richard E, et al. Evaluation of lockdown effect on SARS-CoV-2 dynamics through viral genome quantification in waste water, Greater Paris, France, 5 March to 23 April 2020. Euro Surveill. 2020;25:2000776. https://doi.org/10.2807/1560-7917.ES.2020.25.50.2000776

22. Rimoldi SG, Stefani F, Gigantiello A, Polesello S, Comandatore F, Mileto D, et al. Presence and infectivity of SARS-CoV-2 virus in wastewaters and rivers. Sci Total Environ. 2020;744:140911. https:/ / doi.org/10.1016/ j.scitotenv.2020.140911

23. Nemudryi A, Nemudraia A, Wiegand T, Surya K, Buyukyoruk M, Cicha C, et al. Temporal detection and phylogenetic assessment of SARS-CoV-2 in municipal wastewater. Cell Rep Med. 2020;1:100098. https://doi.org/ 10.1016/j.xcrm.2020.100098

24. 2019-novel coronavirus (2019-nCoV) real-time rRT-PCR panel primers and probes [cited 2020 Jul 23]. https:/ / www. fda.gov/media/134922/download

25. Corman VM, Landt O, Kaiser M, Molenkamp R, Meijer A, Chu DK, et al. Detection of 2019 novel coronavirus (2019-nCoV) by real-time RT-PCR. Euro Surveill. 2020;25:2000045. https:// doi.org/10.2807/1560-7917.ES.2020.25.3.2000045

26. Oude Munnink BB, Nieuwenhuijse DF, Stein M, O'Toole Á, Haverkate M, Mollers M, et al. Rapid SARS-CoV-2 wholegenome sequencing and analysis for informed public health decision-making in the Netherlands. Nat Med. 2020;26:1405-10.

27. Richard M, Kok A, de Meulder D, Bestebroer TM, Lamers MM, Okba NMA, et al. SARS-CoV-2 is transmitted via contact and via the air between ferrets. Nat Commun. 2020;11:3496. https:// doi.org/10.1038/s41467-020-17367-2

28. Martin M. Cutadapt removes adapter sequences from high-throughput sequencing reads. EMBnet J. 2011;17:10-2. https:// doi.org/10.14806/ej.17.1.200

29. Okonechnikov K, Golosova O, Fursov M; UGENE team. Unipro UGENE: a unified bioinformatics toolkit. Bioinformatics. 2012;28:1166-7. https:/ / doi.org/10.1093/ bioinformatics/bts091

30. Oude Munnink BB, Nieuwenhuijse DF, Sikkema RS, Koopmans M. Validating whole genome nanopore sequencing, using Usutu virus as an example. J Vis Exp. 2020;157:e60906. https://doi.org/10.3791/60906

31. Afgan E, Baker D, Batut B, van den Beek M, Bouvier D, Cech $\mathrm{M}$, et al. The Galaxy platform for accessible, reproducible and collaborative biomedical analyses: 2018 update. Nucleic Acids Res. 2018;46(W1):W537-44. https://doi.org/10.1093/nar/gky379

32. Chen S, Zhou Y, Chen Y, Gu J. fastp: an ultra-fast all-inone FASTQ preprocessor. Bioinformatics. 2018;34:i884-90. https://doi.org/10.1093/bioinformatics/bty560

33. Grubaugh ND, Gangavarapu K, Quick J, Matteson NL, De Jesus JG, Main BJ, et al. An amplicon-based sequencing framework for accurately measuring intrahost virus diversity using PrimalSeq and iVar. Genome Biol. 2019;20:8. https://doi.org/10.1186/s13059-018-1618-7

34. Wu F, Zhao S, Yu B, Chen Y-M, Wang W, Song Z-G, et al. A new coronavirus associated with human respiratory disease in China. Nature. 2020;579:265-9. https://doi.org/10.1038/ s41586-020-2008-3 
35. Farkas K, Hillary LS, Malham SK, McDonald JE, Jones DL. Wastewater and public health: the potential of wastewater surveillance for monitoring COVID-19. Curr Opin Environ Sci Health. 2020;17:14-20. https:// doi.org/10.1016/ j.coesh.2020.06.001

36. Michael-Kordatou I, Karaolia P, Fatta-Kassinos D. Sewage analysis as a tool for the COVID-19 pandemic response and management: the urgent need for optimised protocols for SARS-CoV-2 detection and quantification. J Environ Chem Eng. 2020;8:104306. https:// doi.org/10.1016/ j.jece.2020.104306

37. Crits-Christoph A, Kantor RS, Olm MR, Whitney ON, Al-Shayeb B, Lou YC, et al. Genome sequencing of sewage detects regionally prevalent SARS-CoV-2 variants. MBio. 2021;12:e02703-20. https:/ / doi.org/10.1128/mBio.02703-20

38. Mykytyn AZ, Lamers MM, Okba NMA, Breugem TI, Schipper D, van den Doel PB, et al. Susceptibility of rabbits to SARS-CoV-2. Emerg Microbes Infect. 2021;10:1-7. https://doi.org/10.1080/22221751.2020.1868951

39. Oreshkova N, Molenaar RJ, Vreman S, Harders F, Oude Munnink BB, Hakze-van der Honing RW, et al. SARS-CoV-2 infection in farmed minks, the Netherlands, April and May 2020. Euro Surveill. 2020;25:2001005. https:/ / doi.org/10.2807/1560-7917.ES.2020.25.23.2001005

40. Halfmann PJ, Hatta M, Chiba S, Maemura T, Fan S, Takeda M, et al. Transmission of SARS-CoV-2 in domestic cats. N Engl J Med. 2020;383:592-4. https:// doi.org/10.1056/ NEJMc2013400

41. Schlottau K, Rissmann M, Graaf A, Schön J, Sehl J, Wylezich C, et al. SARS-CoV-2 in fruit bats, ferrets, pigs, and chickens: an experimental transmission study. Lancet Microbe. 2020;1:e218-25. https://doi.org/10.1016/ S2666-5247(20)30089-6

42. Shi J, Wen Z, Zhong G, Yang H, Wang C, Huang B, et al. Susceptibility of ferrets, cats, dogs, and other domesticated animals to SARS-coronavirus 2. Science. 2020;368:1016-20. https://doi.org/10.1126/science.abb7015

43. Nieuwenhuijse DF, Oude Munnink BB, Phan MVT, Munk P, Venkatakrishnan S, Aarestrup FM, et al.; Global Sewage Surveillance project consortium. Setting a baseline for global urban virome surveillance in sewage. Sci Rep. 2020;10:13748. https://doi.org/10.1038/s41598-02069869-0

44. Lun JH, Crosbie ND, White PA. Genetic diversity and quantification of human mastadenoviruses in wastewater from Sydney and Melbourne, Australia. Sci Total Environ. 2019;675:305-12. https://doi.org/10.1016/j.scitotenv. 2019.04.162

45. Zhang L, Jackson CB, Mou H, Ojha A, Peng H, Quinlan BD, et al. SARS-CoV-2 spike-protein D614G mutation increases virion spike density and infectivity. Nat Commun. 2020;11:6013. https:// doi.org/10.1038/s41467-020-19808-4

46. Toyoshima $Y$, Nemoto K, Matsumoto S, Nakamura $Y$, Kiyotani K. SARS-CoV-2 genomic variations associated with mortality rate of COVID-19. J Hum Genet. 2020;65:1075-82. https://doi.org/10.1038/s10038-020-0808-9

47. Karamitros T, Papadopoulou G, Bousali M, Mexias A, Tsiodras S, Mentis A. SARS-CoV-2 exhibits intra-host genomic plasticity and low-frequency polymorphic quasispecies. J Clin Virol. 2020;131:104585. https://doi.org/ 10.1016/j.jcv.2020.104585

Address for correspondence: Miranda de Graaf, Department of Viroscience, Wytemaweg 80, 3015CN, Rotterdam, The

Netherlands; email: m.degraaf@erasmusmc.nl

\section{EID Podcast Livestock, Phages, MRSA, and People in Denmark}

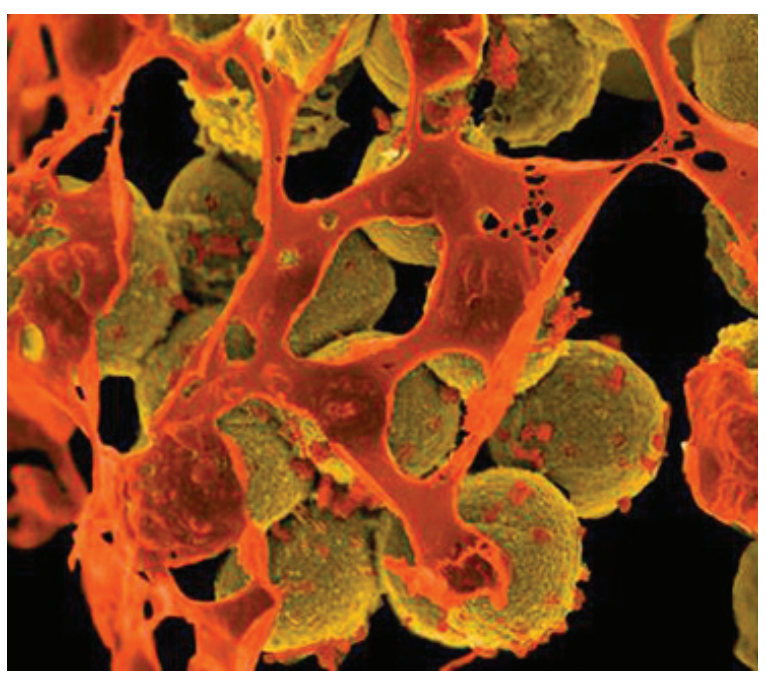

Methicillin-resistant Staphylococcus aureus, better known as MRSA, is often found on human skin. But MRSA can also cause dangerous infections that are resistant to common antimicrobial drugs. Epidemiologists carefully monitor any new mutations or transmission modes that might lead to the spread of this infection.

Approximately 15 years ago, MRSA emerged in livestock. From 2008 to 2018, the proportion of infected pigs in Denmark rocketed from 3.5\% to $90 \%$.

What happened, and what does this mean for human health?

In this EID podcast, Dr. Jesper Larsen, a senior researcher at the Statens Serum Institut, describes the spread of MRSA from livestock to humans.

Visit our website to listen: https://go.usa.gov/x74Jh EMERGING INFECTIOUS DISEASES 